\title{
Correction to: Comparison between percutaneous and open reduction for treating paediatric talar neck fractures
}

\author{
Xu Zhang ${ }^{1} \cdot$ Xinzhong Shao ${ }^{1} \cdot$ Yadong Yu $^{1} \cdot$ Yingzhe Zhang $^{1} \cdot$ Guisheng Zhang ${ }^{1}$ • \\ Dehu Tian ${ }^{1}$
}

Published online: 5 October 2017

(C) SICOT aisbl 2017

Erratum to: International Orthopaedics (SICOT)

https://doi.org/10.1007/s00264-017-3631-y

In the original publication of this paper, the author name was incorrectly presented.

Incorrect presentation: Guizheng Zhang

Correct presentation: Guisheng Zhang

The correct name is presented above.

The original article has been corrected.

The online version of the original article can be found at https://oi.org/ 10.1007/s00264-017-3631-y

Xinzhong Shao

shaoxinzhong@sina.com

Xu Zhang

ahand@sina.com

1 Hand Surgery and Orthopaedics Department, Third Hospital of

Hebei Medical University, Shijiazhuang, Hebei 050051, China 